This document is confidential and is proprietary to the American Chemical Society and its authors. Do not copy or disclose without written permission. If you have received this item in error, notify the sender and delete all copies.

\title{
Electrochemical control of pH in nano-litre volumes
}

\begin{tabular}{|r|l|}
\hline Journal: & Nano Letters \\
\hline Manuscript ID & nl-2017-05054h.R1 \\
\hline Manuscript Type: & Communication \\
\hline Complete List of Authors: & $\begin{array}{l}\text { Balakrishnan, Divya; Luxembourg Institute of Science and Technology, } \\
\text { Material Research and Technology } \\
\text { Lamblin, Guillaume; Luxembourg Institute of Science and Technology, } \\
\text { Material Research and Technology } \\
\text { Thomann, Jean-Sebastien; Luxembourg Institute of Science and } \\
\text { Technology, Materials Research and Technology (MRT) } \\
\text { van den Berg, Albert; University of Twente, MESA Research Institute } \\
\text { Olthuis, Wouter; Universiteit Twente MESA+ } \\
\text { Pascual Garcia, Cesar; Luxembourg Insitute of Science and Technology, } \\
\text { Materials Research and Technlogy }\end{array}$ \\
\hline \hline
\end{tabular}

\section{SCHOLARONE ${ }^{\text {m }}$}

Manuscripts 


\title{
Electrochemical control of $\mathrm{pH}$ in nano-litre volumes
}

\author{
Divya Balakrishnan ${ }^{a, b}$, Guillaume Lamblin ${ }^{a}$, Jean Sebastien Thomann ${ }^{a}$, Albert van den Berg ${ }^{b}$, \\ Wouter Olthuis $^{b}$, César Pascual-Garcia ${ }^{a} *$
}

a. Luxembourg Institute of Science and Technology (LIST), 41 Rue du Brill, L-4422 Belvaux, Luxembourg

b. MESA+ Institute, University of Twente, Drienerlolaan 5, 7522 NB Enschede, Netherlands.

\begin{abstract}
The electrochemical management of the proton concentration in miniaturised dimensions opens the way to control and parallelise multi-step chemical reactions but still it faces many challenges linked to the efficient proton generation and control of their diffusion. Here we present a device operated electrochemically that demonstrates the control of the $\mathrm{pH}$ in a cell of $\sim 140 \mathrm{~nL}$. The device comprises a microfluidic reactor integrated with a hydraulic mechanism that allows the exchange of reagents and the isolation of protons to decrease the effect of their diffusion. We monitored the $\mathrm{pH}$ with a fluorescence marker and calculated the final value from the redox currents. We demonstrate a large $\mathrm{pH}$ amplitude control from neutral $\mathrm{pH}$ 's beyond the fluorescence marker range at $\mathrm{pH}$ 5. Based on the calculations from the faradaic currents, the minimum $\mathrm{pH}$ reached should undergo $\mathrm{pH} \sim 0.9$. The $\mathrm{pH}$ contrast between neutral and acid $\mathrm{pH}$ cells can be maintained during periods longer than 15 minutes with an appropriate design of a diffusion barrier.
\end{abstract}

KEYWORDS: pH control, proton generation, microreactor, proton diffusion, pseudocapacitance. 


\section{INTRODUCTION}

The acidity or proton concentration in aqueous and organic solutions can be used to control a large number of chemical reactions. Some of them, like the assembly of biopolymers ${ }^{[1,2]}$, the control of enzymatic reactions ${ }^{[3,4]}$, the poration of cellular membranes ${ }^{[5]}$ or the self-assembly of mesostructures ${ }^{[6]}$ are fundamental for such important and disruptive applications like the in-situ synthesis in microarrays ${ }^{[7-10]}$, the creation of DNA logic devices ${ }^{[10-12]}$, systematic studies of drug delivery or the fabrication of new functional materials for the healthcare ${ }^{[13]}$. These applications can benefit from multiplexed schemes and the automation of repetitive routines. As it happened with transistors allowing the digital development in the last century, the device miniaturisation and electronic control is the key to achieve the necessary high-throughput to allow a qualitative step forward in the current state of the art of the automatic control of chemical reactions. However, in order to open the door for the implementation of many new applications into practical cases it is necessary to develop the means to automate and miniaturise the control of the $\mathrm{pH}$.

While the effect of the chemical environment on electrodes is widely used for the development of many electrochemical sensors, its reciprocal, namely the use of electrodes to modify the chemical environment is much less studied and quite challenging ${ }^{[14]}$. In particular, the electrochemical control of $\mathrm{pH}$ to implement the automation of chemical reactions has been addressed before typically by the use of proton generation or consumption redox reactions ${ }^{[15-18]}$. However these approaches lack a good control of the $\mathrm{pH}$, lead to the degradation of electrodes and/or the production of side products like hydrogen or oxygen. Many of these reactions are also 
irreversible, blocking the implementation of multi-step processes. In addition, because of the small surface-to-volume ratio these systems usually achieved small $\mathrm{pH}$ changes. More recently, the cyclic control of $\mathrm{pH}$ in macroscopic volumes of few millilitres was demonstrated through the use of gold nano-composites ${ }^{[19]}$ or the use of hydrogen permeable bifacial working electrodes (WEs) ${ }^{[20]}$. Yet the use of these configurations for miniaturised volumes requires modifications able to anticipate the fast diffusion between the electrodes and the reduction reactions of the protons at the counter electrode $(\mathrm{CE})$, the protection of the electrodes that undergo several cycles and the possibility to exchange reagents in a multi-step process.

Here we present and demonstrate a design that takes advantage of the large surface area that can be achieved by microfabrication. For the control of the $\mathrm{pH}$ we use nano-structured electrodes functionalised with redox active self-assembled monolayers (SAM's), which we recently demonstrated to be capable of exchanging protons with small voltage biases in a reversible way for more than hundred cycles ${ }^{[21]}$. The reactor design is integrated in a microfluidic platform that allows addressing the electrodes from an external control. In addition it comprises optical access to monitor the fluorescence of a $\mathrm{pH}$ marker and hydraulic actuation to open and close all the cells that can be miniaturised below $\mathrm{nL}$ volumes while enabling the possibility to exchange reagents for sequential steps. The $\mathrm{pH}$ changes were tracked by a fluorescence marker down to its minimum range at $\mathrm{pH} \sim 5$. From the faradaic currents associated with proton exchange reactions we calculated that the real $\mathrm{pH}$ change was from neutral to $\mathrm{pH} \sim 0.9$. With our experiments we demonstrated the production of protons and the control of their diffusions between cells of $\mathrm{nL}$ volumes. Technologically, the $\mathrm{pH}$ changes within the SNARF range are sufficient to unlock many enzymatic reactions ${ }^{[3,4,9,12]}$, while the more abrupt changes below the SNARF range open the door to improve the yield of organic synthesis of biopolymers like nucleotides or peptides 


\begin{abstract}
[1,2,6-10]. The proton diffusion was controlled using a custom designed diffusion barrier that allowed to validate that the $\mathrm{pH}$ contrast in the electrochemical cells did not significantly vary during more than 15 minutes. The major technological improvements with respect to other configurations in literature are: the demonstration of the large $\mathrm{pH}$ changes confined in $\mathrm{nL}$ volumes, the long lasting stability of the set $\mathrm{pH}$ and the use of immobilised SAM's to protect the electrodes from corrosion.
\end{abstract}

\title{
EXPERIMENTAL
}

\section{A. Design and fabrication of the $\mu$-electrochemical cells.}

The general design of the electrochemical micro-reactor is schematised in fig. 1(a). It consists of a two electrode cell where the WE and the CE electrodes are separated by a diffusion barrier. The electrodes are functionalised with organic molecules able to store or release protons at low applied voltage. Shown in Fig. 1(b), the design may also include a pseudo-reference electrode connected to the WE through a diffusion barrier. The electrodes were platinised to obtain a large surface to area ratio, favourable for the impact of proton exchange reactions in the $\mathrm{pH}$.

The oxidation and reduction of organic aromatic molecules like aniline can be used in a reversible manner to exchange protons with the objective of changing the acidity. These reactions happen at low potentials, which makes them suitable for the control of $\mathrm{pH}$ in an electrochemical cell where other reagents could be present ${ }^{[21-23]}$. Some approaches have used the small $\mathrm{pH}$ changes happening in proximity of the electrodes during the oxidation of aniline to control other chemical processes ${ }^{[6-8,10,13,24]}$, but the fast diffusion of protons in liquids hampers the use of this method to processes that require long reaction times or large $\mathrm{pH}$ amplitudes. In order to overcome, at least partially, these issues electrodes coated with porous materials 
immersed in scavenging solutions were used allowing the synthesis of nucleotide polymers ${ }^{[9]}$. However the remaining proton diffusion and the buffering effect of the electrolyte hampered the efficiency of these reactions that needs to be close to $100 \%$ in order to meet the requirements of commercial applications.

Conceptually, the simplest solution to avoid the proton diffusion may be their physical confinement. Blocking the diffusion of reagents in independent cells has been demonstrated to improve the contrast and the yield of multiplexed peptide and nucleotide reactions in a microfluidic configuration very similar to the one we employ ${ }^{[25,31]}$. However to confine the electrochemical redox reactions involving protons one must take into account that, while they are produced in the oxidation processes at the WE they are also reduced in the CE into hydrogen gas. To overcome this issue one solution is to separate physically the electrodes by diffusion barriers. For the ease of implementation and design we have chosen a linear diffusion barrier made with a long channel in form of serpentine characterised by a length $L$, much longer than their width and height. Migration and diffusion, the two mechanisms that bring protons from the WE to the CE, can be considered in this case one dimensional. Migration may be avoided with a moderate ionic strength (see SI). The use of high ionic strength electrolytes (1 $\mathrm{M} \mathrm{KCl}$ in our case) also decreases the over-potential produced by the current resistivity across the diffusion barrier. The diffusion in one dimension will be ruled by the length of the barrier and the diffusion constant of protons $\left(D=9 \cdot 10^{-5} \mathrm{~cm}^{2} / \mathrm{s}\right)^{[32]}$, which provides the system a characteristic diffusion time $\tau$ in which the diffusion barrier is effective:

$$
\tau=L^{2} / \pi^{2} D \quad \text { Eq. }(1)^{[27]}
$$

Considering the cell of fig. 1 (a) where the of WE and CE are separated by the unidimensional diffusion barrier, redox currents producing only protons and that these will exceed the ones at 
neutral conditions (see SI for the limits of this approximation), for times much smaller than $\tau$, the pH can be approximated from the Faradaic redox currents $\left(I_{r e d o x}\right)$ or the total exchanged charged (Q) as follows:

$$
p H=-\log \left[\frac{I_{\text {redox }} \cdot t}{F \cdot V_{\text {cell }}}\right]=-\log \left[\frac{Q}{F \cdot V_{\text {cell }}}\right] \quad \text { Eq. (2) }
$$

were $t$ is the time during which the current has been applied, $F$ is the Faraday constant and $V_{\text {Cell }}$ is the volume of the cell at the WE. The use of surface functionalised electrodes with redox active self-assembled monolayers (SAMs) like in [21], can be advantageous with respect to the use of aniline in solution because it avoids unnecessary diffusion limited mechanisms and the preservation of the electrodes that may suffer the polymerisation at the surface. In a redox active SAM the maximum charge exchange possible is the number of molecules at the surface (the surface functionalisation, $S)$ multiplied by the total area of the functionalised electrode $\left(A_{W E}\right)$ (and in this case, by the number of electrons exchanged per molecule). Eq. 2 can then be simplified considering also that the volume of the cell is $A_{W E}$ times its height $h$ to find what the minimum achievable $\mathrm{pH}$ is:

$$
p H=-\log \frac{s \cdot A_{W E}}{h \cdot A_{W E}}=-\log \frac{s}{h} \quad \text { Eq. (3). }
$$

Thus, in order to favour large acidic amplitudes one must decrease as much as possible the height of the cell and increase the surface functionalisation. Nano-structuration of electrodes achieved by methods like platinisation ${ }^{[33]}$ can increase the electrochemical surface area and the effective $S$.

Figure 1 (b) shows the design used in this article for the implementation of the electrochemical cell where the WE was connected with a serpentine barrier to the CE. The design includes also a third cell with a gold pseudo-reference electrode that we used to have a better control of the 
redox reactions, and which was also connected by a serpentine diffusion barrier to the working electrode to be protected from the $\mathrm{pH}$ variations. The electrodes occupied a total area of $1 \mathrm{~cm}$ diameter, in which diffusion barriers longer than $2 \mathrm{~cm}$ could be introduced. The width of the diffusion barrier was $100 \mu \mathrm{m}$ and the height delimited by the dielectric spacer (SU8 epoxy in our case) was $3.5 \mu \mathrm{m}$ before the platinisation. Different chips were fabricated on top of $100 \mu \mathrm{m}$ thermally grown oxide. Data in this article come from three of the samples studied. The fabrication steps are schematically detailed in the SI fig S1. The chips were fabricated by performing first the definition of the electrodes by optical lithography, then they were e-beam evaporated using a $3 \mathrm{~nm}$ Ti adhesion layer and $50 \mathrm{~nm}$ deposition of gold. The spacer barriers were defined using also optical lithography on the epoxy resist (SU-8). WE and CE were platinised following standard recipes ${ }^{[33]}$ cycling them in a solution of $40 \mathrm{mM}$ of chloroplatinic acid and $100 \mu \mathrm{M}$ of lead acetate between -0.2 and $0.6 \mathrm{~V}$ against a $\mathrm{Ag} / \mathrm{AgCl}$ reference electrode for 4 cycles at a scan-rate of $100 \mathrm{mV} / \mathrm{s}$. After the platinisation the sample was rinsed in water and ethanol, and then cleaned under UV ozone lamp. This provided the electrodes more surface area, and further decreased the effective height of the chip (see more on supplementary information). Finally the functionalisation of electrodes was achieved by immersion of the chip in $0.5 \mathrm{mM} 4$ amino-thiol-phenol (4ATP) solution in ethanol overnight functionalising all three electrodes. No particular protection was used for the pseudo-reference electrode. The chip was thoroughly rinsed with ethanol and prepared for experiments into the microfluidic platform.

\section{B. $\mu$-fluidic platform and experimental setup.}

Figure 1 (c) shows the schematic configuration of our microfluidic platform that consists in two frames to hold the chip, which is sealed with an elastic O-ring that allows two positions (pictures shown in SI fig S5). In the opened position the O-ring pushes down the exchangeable chip 
allowing $\mathrm{a} \sim 60 \mu \mathrm{m}$ gap between the chip and the top optical access to allow the cell filling and the exchange of fluids. The chip can be pushed up in contact with a glass by a hydraulic actuator placed in the bottom frame to reproduce the configuration shown in fig. 1 (a) and (c) where the cells are isolated from the fluidic channel and connected only through diffusion barriers. The glass may be used as optical access to track fluorescence changes in the cells. This configuration does not follow the normal common paradigm of "lab on a chip" where normal valves and actuators are implemented into channels but it can be used in the future to include several cells allowing the parallelisation of several chemical reactions using a single hydraulic actuation. However the microfluidic filling of the cell may present some issues due to the small dimensions of the channel and the large area of the chip ${ }^{[25]}$. To overcome these problems vacuum was applied between the chip and the top glass before the filling. The chip is electrically contacted through springs included in the top frame and connected to a switching box to allow the electrodes to be grounded or connected to instrumentation. The microfluidic platform was placed in the experimental set-up shown in fig. 1 (d). The electric signal was controlled by a Solartron Modulab XM Pstat 1MS/s potentiostat using two and three electrode configurations. The platform allows also optical inspection through the top glass window closing the cells.

The measurement of $\mathrm{pH}$ in $\mathrm{nL}$ volumes is a challenge in itself. Electrochemical measurement of $\mathrm{pH}$ requires the integration of reference electrodes that consume valuable footprint, and which can be difficult to incorporate. Only sensors like nanowires ${ }^{[26,27]}$ would be suitable with the design of our small cells. Optical methods do not have an impact in the footprint. Plasmonic devices using the enhancement of fluorophores or Raman signals associated to nanoparticles can report high intensities ${ }^{[28,29]}$ but they require a long development for standardisation. Fluorescence sensors are an effective way to monitor the $\mathrm{pH}^{[30]}$ if the signal is sufficient and the 
fluorophore does not interact with the electrodes. The intensity of a fluorescence peak associated with a mini-band created by the electronic structure of an aromatic component of the molecule changes with the degree of protonation of some of the radicals. Fluorescein is one of the typical dyes with a high fluorescence yield used to measure the $\mathrm{pH}$ having one peak that, once calibrated, can report the $\mathrm{pH}$ value. The problem with single peak $\mathrm{pH}$ reporters is that the peak intensity can also change due to factors like photo-bleaching, aging or simply a change in the optical alignment.

The fluorescence dye carboxy semi-naphthorhodafluors (carboxy SNARFs) instead has two bands at 590 and $650 \mathrm{~nm}$, which ratio can be used to track the $\mathrm{pH}$ in a range approximately from 8 to $5^{[19]}$. The electrolyte filling of the micro-reactor consisted of $1 \mathrm{M} \mathrm{KCl}$, with the addition of $0.5 \mu \mathrm{M}$ of SNARF. This dye was previously used with the same concentration to measure electro generated protons ${ }^{[19]}$. These experiments and also ours (see the description of the experiments of electro-polymerisation in SI) proved that the $\mathrm{pH}$ measurements were not affected by interactions of the fluorophore with the electrodes. Figure 1 (e) shows the calibration of the SNARF fluorescence with $\mathrm{pH}$ using the relative intensity of the peaks at $530 \mathrm{~nm}$ and $580 \mathrm{~nm}$ excitation and also the intensity of the $650 \mathrm{~nm}$ line normalised to the peak intensity at $\mathrm{pH} 7.3$. The inset shows representative spectra of the fluorescence using the $530 \mathrm{~nm}$ excitation (dotted lines). The $590 \mathrm{~nm}$ peak is stronger at acidic $\mathrm{pHs}$ while the $650 \mathrm{~nm}$ is dominant at neutral $\mathrm{pH}$ 's. In order to increase the fluorescence signal the light collected by the objective was sent into an optical fibre using collecting lenses, and then sent to a wide range spectrometer FLAME-S-VIS-NIR. With this configuration the illuminated/collected region had a radius of $\sim 63.5 \mu \mathrm{m}$ in which the excitation power $(1.3 \mathrm{~mW})$ was distributed and averaged over a large number of molecules $\left(\sim 10^{7}\right.$ molecules) avoiding photo-bleaching and limiting the thermal heating (details in SI). All the 
spectra in the article, unless otherwise specified were integrated for $1 \mathrm{~s}$, to allow monitoring real time the $\mathrm{pH}$ changes. Due to the low integration time and the low power used we were forced to excite at $580 \mathrm{~nm}$ close to the maximum of absorption of SNARF (grey line in the inset of figure 1 (e)) in order to maximise the fluorescence efficiency. To avoid the overlap of the signal from the excitation diode and the stray light, we used a dichroic mirror CROMA-49017 with an excitation window between 540 and $580 \mathrm{~nm}$ and with a low band pass filter at $590 \mathrm{~nm}$ for collection. The inset of figure 1(e) shows also the comparison of the fluorescence spectra with the $530 \mathrm{~nm}$ excitation line and the 580 through the dichroic mirror (solid and dotted lines respectively). The spectra using both excitations were fit to two-Gaussian peak functions, with fixed peak centre and width. Even the spectra through the dichroic mirror were cut close to the centre of the $590 \mathrm{~nm}$ peak it was possible to fit them with enough accuracy, and both calibrations provided $650 \mathrm{~nm} / 590 \mathrm{~nm}$ peaks resulting in similar values (solid and open triangles for the excitation at $530 \mathrm{~nm}$ and $580 \mathrm{~nm}$ respectively). The only difference we observed was slightly higher values of the $590 \mathrm{~nm}$ peaks for the $580 \mathrm{~nm}$ excitation, which can be explained by a closer resonance of the illumination wavelength with respect to that band. The red dots in figure 1 (e)

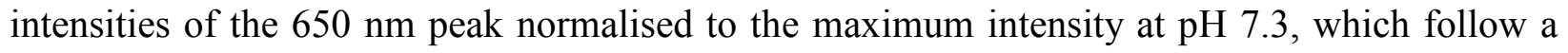
similar behaviour of the ratio of the two peaks. Thus the spectrum at $\mathrm{pH} 7.3$ can be used to assess the initial $\mathrm{pH}$. The normalisation of the peak at $650 \mathrm{~nm}$ to the peak intensity at 7.3 provides then a calibration independent of external factors like the alignment. Since the $\mathrm{pH}$ in our device could go below the $\mathrm{pH}$ range of the SNARF in the article we show directly the fluorescence of the SNARF or the normalised peak intensity instead of the $\mathrm{pH}$ calibration.

RESULTS AND DISCUSSION

\section{A. Electrochemical control of $\mathrm{pH}$ in confined cells}


The electro-polymerisation of 4ATP has been well studied on different articles including our own ${ }^{[21-23]}$. The resulting molecules are the dimers depicted in the inset of Fig. 2 (a) that support the quasireversible redox exchange reactions involving the exchange of 2 protons and 2 electrons. We performed the electro-polymerisation of the 4ATP layers functionalising the platinised electrodes with the cell opened and tracking the $\mathrm{pH}$ (details shown in the SI). After the polymerisation the electrolyte of the cell was exchanged to recover neutral $\mathrm{pH}$ and equilibrium conditions. The cell was then closed leaving the WE communicated only through diffusion barriers to the counter and the pseudo-reference electrodes, and connected to our potentiostat as shown in figure 2 (b). The cyclic voltammogram of the polymerised 4ATP is shown in Fig 2 (a). The $\mathrm{CV}$ potential was narrowed with respect to the polymerisation process between $\pm 0.75 \mathrm{~V}$, using a scan-rate of $50 \mathrm{mV} / \mathrm{s}$. The characteristic quasi-reversible oxidation and reduction peaks attributed to proton exchange reactions of the polymerised 4ATP were observed at $\sim 0.35$ and $\sim$ $0.3 \mathrm{~V}$ respectively. It is noteworthy highlighting that during the electropolymerisation that we did in the chamber (see SI) there was no oxidation peak at $0.35 \mathrm{~V}$ during the first cycle before the electropolymerisation, indicating that only the polymerised molecules lead to reversible proton exchange reactions and that there is little or no contribution from the electrolyte (that contained SNARF). A change of the redox potentials is observed in our platinised electrodes with respect to the reactions_on flat gold ${ }^{[21]}$, which is the same as the one observed with the opened cell (see more experimental data from the electropolymerisation in SI), which shows that it is not due to the IR drop at the diffusion barrier, but rather to the different material of the electrode (nanostructured Pt in our case). The oxidation of the first cycle is a particular case because in this cycle the current is limited because at the CE there are no dimers of 4ATP that can reduce. The rest of the cycles in the voltammogram overlap, indicating that the proton 
distribution from the equilibrium at the resting point reaches a steady state after the first cycle, with no further contributions from electro-polymerisation and that the number of protons in the system is constant. The reversibility of the CVs is also an indication that the electrodes do not suffer degradation and that the redox reactions are associated to reversible proton exchange reactions. Figures 2 (c) and (d) show the measured current and applied voltage time evolution, respectively. Cycles 2, 5 and 8 are highlighted in red, green_and blue respectively and the rise and fall voltage of the cycles are depicted with light and darker colours respectively. The colour code on the cycles is maintained in all the figures including the representation of the fluorescence spectra.

Figures 2 (e), (f) and (g) show the sequence of SNARF fluorescence spectra extracted during the cycles 2, 5 and 8 with a vertical offset corresponding to equally spaced voltage differences during each cycle. The spectra corresponding to the positive and negative sweep rates and are indicated with light and dark tonalities matching the colours of figs. 2 (c) and (d). The left and right panels on each graph figure report the spectra taken at the WE and CE respectively. They were acquired first focusing the objective on top of the WE and then the experiment was repeated collecting the signal from the CE. Several series of $10 \mathrm{CV}$ cycles showed to be equivalent after several experiments, so the spectra from the $\mathrm{CE}$ and WE were grouped according the time and voltage with respect to the open circuit before applying the driving voltage. The spectra just above the green dotted line correspond to the ones at the voltage indicated with the green arrow in Fig 2 (a) just before the oxidation process. These spectra show the maximum intensity dominated in all the cycles at the WE only by the peak at $650 \mathrm{~nm}$ indicating that the $\mathrm{pH}$ in that electrode was close to the initial neutral conditions before the oxidation that were used as reference. The behaviour is opposite at the CE where $650 \mathrm{~nm}$ 
fluorescence was completely quenched beyond the SNARF range, indicating that from cycle $2(\mathrm{t}$ $=50 \mathrm{~s}$ ) the polymerised 4ATP had released the protons to convert the cell environment of the CE into an acidic medium below $\mathrm{pH} 5$ by the release of protons. After the oxidation of the 4ATP at the WE the SNARF fluorescence decreases indicating that the $\mathrm{pH}$ becomes lower in this cell and the opposite happens at the $\mathrm{CE}$ where the rise in the fluorescence signal is an indication of the increase of the $\mathrm{pH}$ because the protons in solution are recovered by the 4ATP. The comparison among the spectra at figs. 2 (e) to (g) show that the overall intensity of the SNARF fluorescence increases from cycle 2 to 8 both in the WE and CE cells. This increase of fluorescence reflects an increase of the average $\mathrm{pH}$ during the cycles and thus it is an indication that the number of protons is decreasing in the system. This can be explained because when the electrodes are negatively biased the protons may get reduced into molecular hydrogen $\mathrm{H}_{2}$, more probably at gaps where 4ATP did not reach to cover the metal, and thus decreasing the total number of protons in the system ${ }^{*}$. However, we did not observed any significant degradation of the 4ATP redox reactions on the $\mathrm{CV}$ and we always obtained the quenching of the fluorescence signal. After each 10 cycles of each experiment, the $\mathrm{pH}$ and the fluorescence could be recovered for several experiments on both WE and CE by exchanging the electrolyte by a fresh one. Each chip could be used for experiments lasting at least one day.

The SNARF fluorescence could track the $\mathrm{pH}$ down to $\mathrm{pH}$ 5. Below the final $\mathrm{pH}$ can be derived using equation 2 since the reversible electrochemical processes in our system are only associated to the proton exchange reactions. Figure 2 (a) shows the overlap of the 10 different cycles studied in this figure, and the hatched area is the area corresponding to the 4ATP oxidation. The amount of charge associated to these faradaic currents is equivalent to the amount of protons released to the electrolyte and it can be used to calculate the total $\mathrm{pH}$ in the cell of the WE (eq. 
2). This was possible in the case of the CV because the shape of the curves allows to distinguish the faradaic currents from the capacitive and resistive ones, but in other experiments the faradaic and non-faradaic currents could not be distinguished. The resulting charge exchanged was $\sim 1.7$ $\mathrm{mC}$ (see calculations in SI). We also consider that the time of each cycle is much smaller than the diffusion time through the barrier connecting the electrodes, and thus the produced protons were distributed only in the cell of the WE. Considering that the initial $\mathrm{pH}$ of the electrolyte was $\sim 7.4$ and that the total volume of the WE cell was $\sim 140 \_\mathrm{nL}$, the maximum proton molarity was $\sim 0.13 \mathrm{M}$, thus the final $\mathrm{pH}$ was $\sim 0.9$. To calculate the volume of the cell we have considered the initial height of the cell before platinisation, since this material is porous and the decrease in volume is difficult to measure (see details in SI). Thus the $\mathrm{pH}$ reported value above is just an upper limit, but this amplitude is at least $\sim 5 \mathrm{pH}$ units, which is enough to control many chemical reactions used in purposes like the ones already cited ${ }^{[1-6]}$.

Figure 2 (h) shows a summary representation of the acid control in the cells. In the middle cartoon the cell status before oxidation is represented with the WE in red for the more basic conditions. In such state the 4ATP layer is reduced and works as a proton reservoir. Meanwhile in the CE the 4ATP is oxidised and the protons are in solution producing an acidic environment (represented in green in the cartoons). The acid concentration in the different cells can be reversed driving the system with voltage or current as represented on the top cartoon, which is the cell status after the oxidation peak. Since the system was driven out of equilibrium starting from a neutral electrolyte, the addition of protons from the 4ATP induces a cell status during the cyclic voltammetry between $\sim 0.6$ and $\sim-0.6 \mathrm{~V}$ where both cells are acid and where the protons in the system are shared between both cells and not stored in one of the WE or CE (including the 
bottom two spectra below the dotted line in figs $2 €$ to $(\mathrm{g}))$. This situation is represented by the bottom cartoon in fig 2 (h) with both cells are depicted with the same colour.

To use the $\mathrm{pH}$ control in an efficient way it is necessary to keep the $\mathrm{pH}$ value constant over time because many chemical reactions are slower than the proton diffusion time. With the cell open, with no current inducing exchange of protons between the electrolyte and the electrodes the $\mathrm{pH}$ in the WE evolves to an equilibrium with the rest of the cell losing the control of the acidity in 10 $\mathrm{s}$ (see SI fig 7 (d) that reports the $\mathrm{pH}$ behaviour during the electropolymerisation with the opened cell). The micro-reactor built-in a $2 \mathrm{~cm}$ diffusion barrier where the diffusion time $\tau$ from the WE to the $\mathrm{CE}$ should be $\sim 75$ minutes (eq. 1). Figure 3 (a) shows the fluorescence spectra after oxidation at the WE right after imposing open circuit conditions (red) and 15 minutes later (blue) with the cell closed to avoid diffusion. The spectra correspond to the same extremes of the $\mathrm{pH}$ shown in figure 2 and they were integrated for $10 \mathrm{~s}$ instead of 1 , which resulted in a lower noise background spectrum shown in black. Figure 3 (b), shows the corresponding spectra at the CE for the same time lapses. The spectra were vertically shifted (the red and blue lines), other ways they would superimpose almost perfectly. In fig. 3 (a), the difference of the red and blue spectra with the background in black show clearly the existence of the peak at $590 \mathrm{~nm}$, which was cut by the band-pass filter of the dichroic mirror. This maximum of fluorescence is the characteristic shape of SNARF when the $\mathrm{pH}$ is below its range in acidic conditions (fig 1 (e) and the comparison with the spectra SI fig S7 (b)). In fig. 3 (b) the fluorescence after current and 15 minutes later are dominated by the $650 \mathrm{~nm}$ peak characteristic of the basic/neutral conditions, reproduced in Figure 1 (e) for $\mathrm{pH}$ 7.3. There were no significant differences between the spectra after the end of the current control and 15 minutes later with open circuit conditions, indicating 
that the $\mathrm{pH}$ was constant in the cell during this time, even with the basic conditions where the SNARF fluorescence is more sensitive to $\mathrm{pH}$ variations.

The proton confinement not only improves the time in which the $\mathrm{pH}$ is stable, but also the amplitude of the $\mathrm{pH}$ that can be achieved because the produced hydrogen ions are distributed in smaller volumes. In figure 4, the applied voltage (in black) and the measured current (in red) is shown as function of time corresponding to the second cycle of CV's for the open and closed cell configurations (figs 4 (a) and (b) respectively). The voltage was narrowed between 0.5 and -0.2 $\mathrm{V}$, which allowed us to control the $\mathrm{pH}$ change within the SNARF range before the fluorescence was completely quenched, but which did not allow to distinguish the faradaic from the nonfaradaic currents to make a $\mathrm{pH}$ calculation. Figures 4 (c) and (d) show nevertheless the corresponding SNARF fluorescence peak intensity collected right at the top of the WE for the open and closed configurations respectively. The existence of redox reactions can be noticed by the non-linear behaviour of the currents and the changes of $\mathrm{pH}$ sensed by the SNARF fluorescence. As observed during the polymerisation experiments (see SI), the $\mathrm{pH}$ changes can also be detected with the cell opened even if the WE and CE were in contact through the electrolyte because the detection was close to the electrode and the focus depth of the microscope is sufficiently small $(1 \mu \mathrm{m})$. However the amplitude of this $\mathrm{pH}$ change is limited to $10 \%$ of the maximum peak fluorescence (fig. 4 (c)) associated to a $\Delta \mathrm{pH}$ of less than $\sim 0.4$ units (fig. 1(e)). This change was even possible due to the small height of the channel compared to the large area of the electrodes, but also because the focus of the microscope was on top of the surface of the electrodes. On the other hand, within the same voltage range the fluorescence quenching with the cell closed could be observed to decrease down to $\sim 60 \%$ of the original maximum peak fluorescence (fig. 4 (d), associated to a $\Delta \mathrm{pH}$ of $\sim 1.4$ units. The increase of the 
amplitude change in the peak fluorescence is a proof of the increase of the $\mathrm{pH}$ change with the closed cell configuration.

\section{B. Control of the pH with a 2 electrode configuration.}

The implementation of reference electrodes in microfluidic devices is a cumbersome task. Equation 2 describes the $\mathrm{pH}$ in the cell as a function of the redox (Faradaic) currents that can be implemented with a two electrode configuration like the one shown in the inset of fig. 5 (a). Yet eq. 2 does not consider the kinetics of the system that is influenced by both the electrochemical Faradaic and the capacitive non-Faradaic components. In addition to the double layer capacitance, the diamine functionalities contribute to the capacitance by storing energy on the redox reactions that exchange electrons (pseudo-capacitance) ${ }^{[34-37]}$. The pseudocapacitance may significantly increase the overall capacitance of the amidized large surface area electrodes and influence the charging and discharging behaviour ${ }^{[29]}$. Figure 5 (a) shows applied square current pulses (in black) of $\pm 4 \mathrm{~mA}$ of amplitude, $2 \mathrm{~s}$ of excitation time and $10 \mathrm{~s}$ of resting time and the corresponding voltage response (in red). They were applied after $5 \mathrm{~s}$ of resting time with the system in equilibrium (open circuit and fresh electrolyte). During the application of the current, the voltage rises non-linearly due to the contribution of the IR drop produced by redox currents and the capacitance of the system followed by a decrease similar to discharge, but with no current flowing from the electrodes to the potentiostat. The normalised peak fluorescence acquired during the current excitations is shown in fig 5 (b). Since the cell is very small, the diffusion of protons is much faster than the integration time of each spectrum so the fluorescence indicates the $\mathrm{pH}$ variations in the cells that depends on the changes of the number of reduced or oxidized groups. A delay with respect to the applied current is observed that follows the 
charging/discharging evolution of the voltage. To interpret this delay and the fact that the $\mathrm{pH}$ continues to lower even if there are no currents in the circuit, we take into consideration that the electron transfer rate of the redox reactions, which we measured in our previous article, is $\sim 0.6 \mathrm{~s}$ [21], which is in the order of the on-time of the current pulse. Thus not all the molecules react during the large current pulse imposed and the built up potential, which is discharged through the redox reactions that we observe with a delay. This process can be described as a combination of a pseudo-capacitance that stores charge originated from the electron transfer between the metal and the benzenoid diamine molecules and a battery-like behaviour that yields a flat discharge plateau $^{[38]}$. Because of the discharging the system does not yield as a good capacitor, but the device shows the capability to study the electrochemical process with the advantage of being able to follow the proton exchange reactions with the $\mathrm{pH}$ probes.

Also noticeable is the difference of the $\mathrm{pH}$ amplitude between the first pulse and the others. As seen in fig. 2 (a), during the first cycle the oxidation reactions are limited because on the counter electrode all the amines are reduced, so they cannot accept protons to balance the oxidation reactions. After the second negative pulse, the system achieves a steady state, where the more basic and more acidic environments alternate between the oxidation and reduction of the working and counter electrodes respectively as explained in fig $2(\mathrm{~h})$. This dependence on the polymerised 4ATP dimers is another evidence that the hydrolysis or other components in the electrolyte are not_responsible for the $\mathrm{pH}$ changes.

We repeated these experiments with current control on the same chip for nine times with different current amplitudes and duty cycles, with always the same behaviour. Complete quenching of the fluorescence could be achieve at large currents (see SI). No signs of fatigue or degradation of the electrodes were observed, which indicates that regardless the high voltages 
measured between the electrodes the polymerised molecules were preserved. Neither did we observed any bubbles on the electrodes, thus we also exclude the electrolysis of water, probably due to the protecting effect of the 4ATP monolayer.

\section{CONCLUSIONS}

We have presented the design of an electrochemical device to control the $\mathrm{pH}$ in miniaturised sytems. The pH is changed using proton exchange reactions, which are produced by quasireversible redox states of polymerised 4ATP molecules immobilised on top of nanostructured electrodes. Compared to other methods, this enhances the reversibility of the reactions with respect to other more corrosive processes ${ }^{[15-18]}$, prevents modification of the electrodes by further polymerisations ${ }^{[7-10]}$, and avoids diffusion limited mechanisms ${ }^{[6-8,10,13]}$. It also allows the possibility to isolate the electrodes in the future to avoid interferences (using for example with proton membranes), although we have not observed any interference from the components of the electrolyte that we have used. Using a cell of a few micrometres high allowed a large impact on the $\mathrm{pH}$ of the occurring redox reactions. We were able to track the $\mathrm{pH}$ changes in a range down to the quenching of our fluorescence marker at 5 and we calculated that the $\mathrm{pH}$ in the cell could reach extremely low values down to $\sim 0.9$.

The effect of the proton diffusion is controlled by a diffusion barrier that can be tailored to maintain a stable $\mathrm{pH}$ in a cell for a time depending on the requirements of the chemical control needed. The comparison of the $\mathrm{pH}$ changes between the open and closed cell configurations showed that the confinement of protons increases the amplitude of the $\mathrm{pH}$ variations and provides a stable $\mathrm{pH}$ for a relatively long time. In our case we proved that the $\mathrm{pH}$ did not change during 15 minutes using a linear diffusion barrier of $2 \mathrm{~cm}$ inserted between electrodes that were 
separated by only $2 \mathrm{~mm}$. The open cell configuration is analogue to the one used in previous articles where they used the $\mathrm{pH}$ changes of aniline to control chemical reactions ${ }^{[6-8,10,13,24]}$. The chemical yield in these applications could improve with a closed cell configuration by benefiting of a higher swing in $\mathrm{pH}$ and longer $\mathrm{pH}$ stability.

The integrated design of the electrochemical cell with microfluidics using a hydraulic actuation allows the exchange of reagents that we used to regenerate the cell for each different experiment by exchanging the liquid with neutral $\mathrm{pH}$. This configuration is compatible with miniaturisation. Indeed the $\mathrm{pH}$ amplitude swing depends only on the height of the cell, and not on its lateral dimensions. The biggest constrain of the design we present relies on the relation of the length of the diffusion barrier and the time in which the $\mathrm{pH}$ is needed to be kept constant, according to eq. 1. To alleviate this issue in the future the diffusion barrier could be substituted by porous materials. Nevertheless the 1D design makes it easy to predict the diffusion time and to tailor the diffusion barrier depending of the chemical reactions that need to be controlled. The microfluidic configuration that we have shown can support different cells in the same channel, thus combined with the miniaturisation of the cells it can be used to multiplex different chemical reactions. Since the cell is compatible with organic electrolytes this device can be used in the future for applications like peptide or nucleotide synthesis that can benefit from the combined effect of the improved $\mathrm{pH}$ control and the decrease of importance of the role of the diffusion of reagents ${ }^{[24,}$ 25].

The integration of reference electrodes in miniaturised devices is a challenging task. The $\mathrm{pH}$ in our case can be conveniently controlled with current using a two electrode configuration, though for the control of the charging/discharging behaviour of the redox reactions the kinetics of the pseudocapacitance associated to the amine redox sites should be taken into account. The $\mathrm{pH}$ 
variations observed during the resting time after the current pulses are to our knowledge the first direct observation of the pseudo-capacitance discharge. The redox mechanism for the capacitance characteristics of the amino-functionalized sites still deserve a deeper investigation, however we show that the configuration of our reactor can be used to track the redox reactions and study the effect of the pseudocapacitance on electrodes employed in other applications like batteries or supercapacitors ${ }^{[34-37]}$.

In summary, we have presented a device aiming for the future miniaturisation and multiplex of chemical reactions with a large electrochemical control of the $\mathrm{pH}$.

CONFLICTS OF INTEREST

The authors declare they have no conflicts of interest to publish this investigation. 


\section{FIGURES}
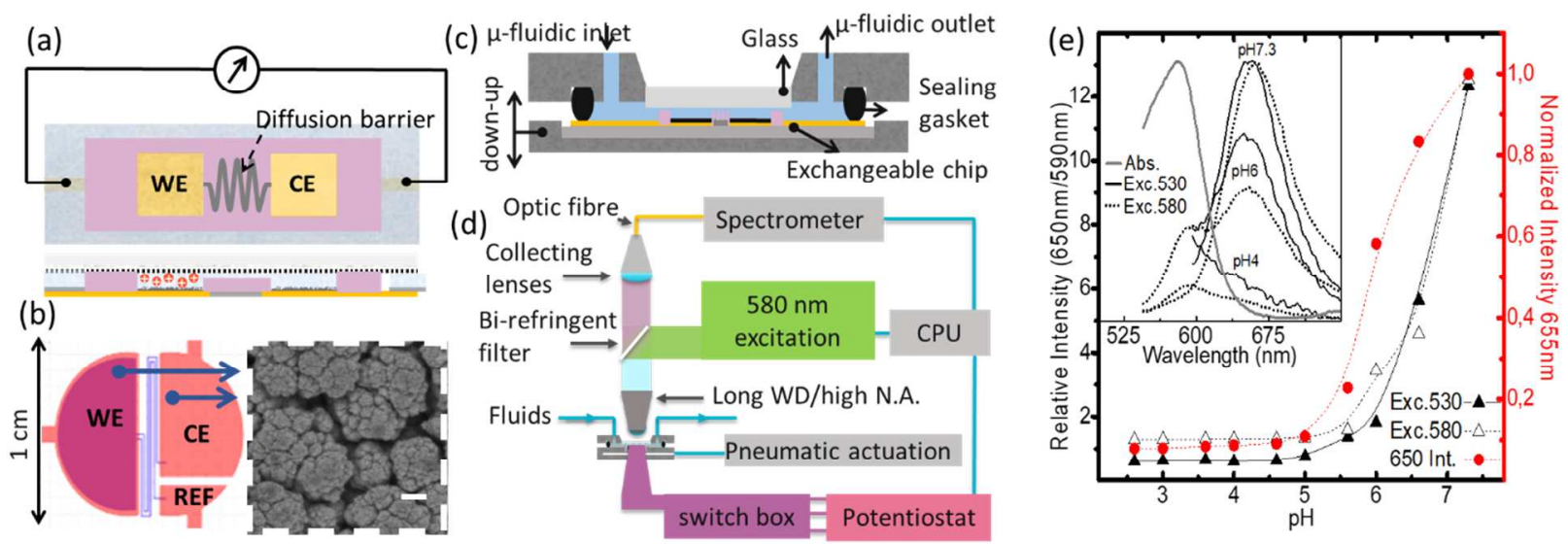

Figure 1: (a) Schematic representation of a simple electrochemical cell able to control the $\mathrm{pH}$ in miniaturised environments. (b) Design of the microfluidic cell including a pseudo-reference electrode and SEM picture of a small region representative of the nanostructured platinised electrodes at the WE and CE (scale bar is $100 \mathrm{~nm}$ ). (c) Schematic representation of the $\mu$-fluidic platform holding the exchangeable chip holding the cells schematised in (b) (See fabrication process of the chip in S.I. fig S1). (d) Schematic representation of the experimental setup used to control and monitor the $\mathrm{pH}$ changes in the microfluidic platform. (e) Calibration of SNARF fluorescence. The relative intensity of the $655 \mathrm{~nm}$ and $580 \mathrm{~nm}$ peaks are shown with closed and open black triangles for the excitation lines of 530 and $580 \mathrm{~nm}$ respectively as a function of the $\mathrm{pH}$. The normalised intensity of the $650 \mathrm{~nm}$ peak as function of the $\mathrm{pH}$ is also shown in red crossed circles. The inset shows the absorption peak at pH 7.3 (grey solid line) and the fluorescence spectra at representative $\mathrm{pH}^{\prime} \mathrm{s}(7.3,6$ and 4) using the excitation lines at $532 \mathrm{~nm}$ and $580 \mathrm{~nm}$ (solid and dotted black lines respectively). 

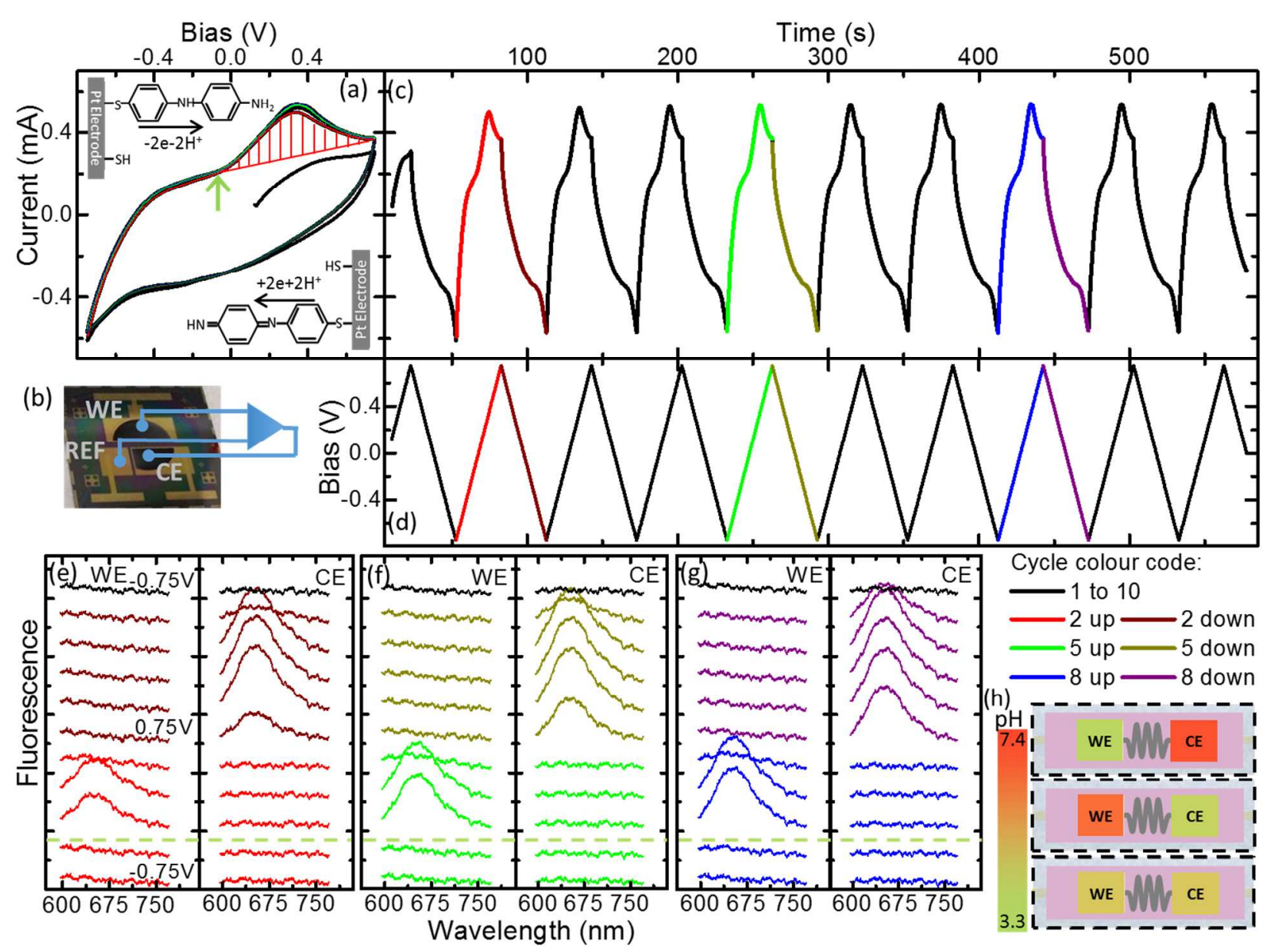

Figure 2 (a) CV of 10 cycles corresponding to the reversible proton exchange reactions of polymerised 4ATP done in a closed cell configuration. The inset shows schematically the 4ATP dimers that produce the oxidation (top to bottom dimer) and reduction (bottom to top dimer) reactions involving the exchange of two protons and two electrons (b) Schematic representation of the chip connections to the potentiostat. (c) and (d) current and voltage vs time during the $\mathrm{CV}$ as a function of time, respectively. Cycles 2, 5 and 8 are highlighted in red, green and blue colour respectively and the rise and fall voltage branches of the cycles are depicted with light and darker colours respectively. The colour code on the cycles is maintained on (e), (f) and (g) where the sequence of SNARF fluorescence spectra acquired at the WE and 
CE (left and right panels on each) are shown. A vertical offset is introduced for each spectrum corresponding to the acquisition at equal voltage differences. The colour changes from light to dark tonalities indicate the voltage sweep is reversed. The arrow in (a) indicates the voltage before the oxidation that corresponds to the spectra just above the green dotted line in (e) to (g). The sequence progresses clockwise. (h) shows a schematic representation of the acid. In the middle cartoon the state before oxidation is shown and the WE is basic (represented with red colour) and the CE is acid (represented by green). After the oxidation (top cartoon) at the WE the $\mathrm{pH}$ becomes acid and opposite in the CE. Since the system is brought from neutral conditions to acid conditions there is a state around $0.6 \mathrm{~V}$ where both cells are acid (Bottom

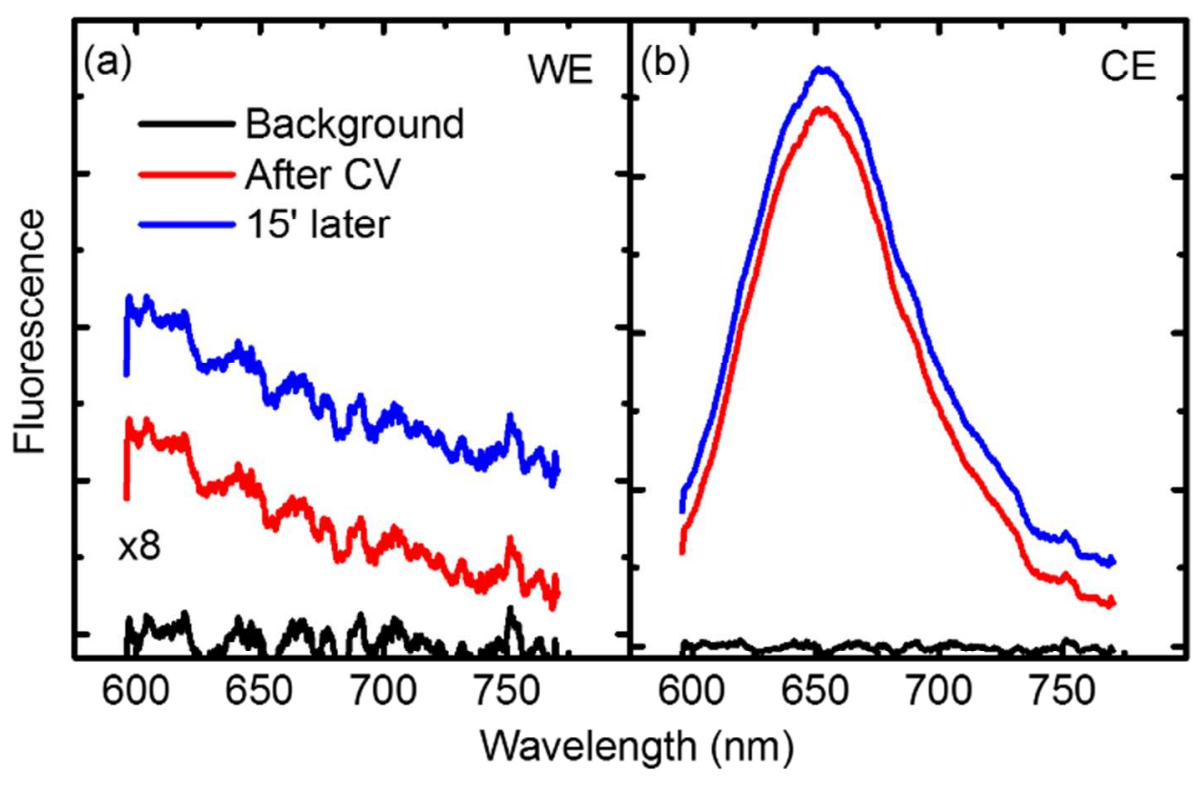

cartoon).

Figure 3 (a) background spectrum (black) and SNARF fluorescence spectra right after an oxidation at the WE (red) and 15 minutes later of open circuit conditions (blue). (b) Equivalent 
spectra obtained at the CE. Spectra were vertically shifted to avoid the overlap of the red and blue spectra. Spectra in (a) are shown with a $8 \mathrm{X}$ amplification of the signal respect to (b).

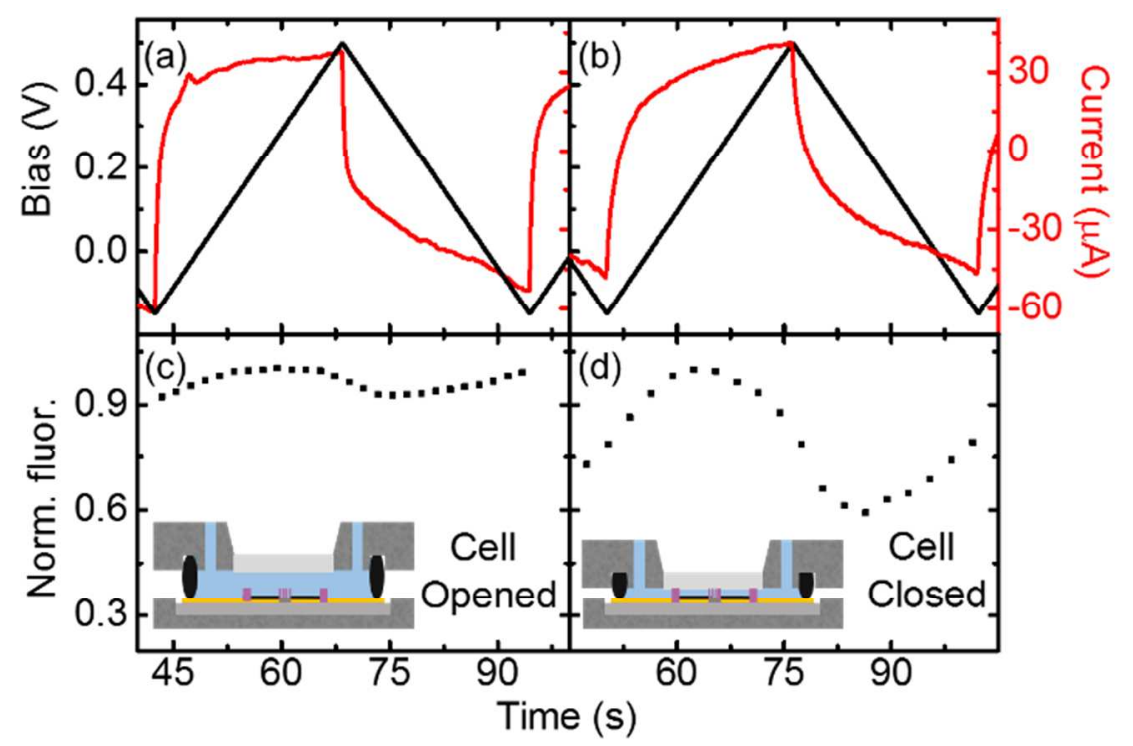

Figure 4 (a) and (b) Voltage bias (in black) and current response (in red) corresponding to the second cycle of a CV narrowed between 0.5 and $-0.2 \mathrm{~V}$ as function of time with the cell opened and close respectively. (c) and (d) corresponding SNARF peak fluorescence intensity at the WE. Vertical scales are common through all the figure.

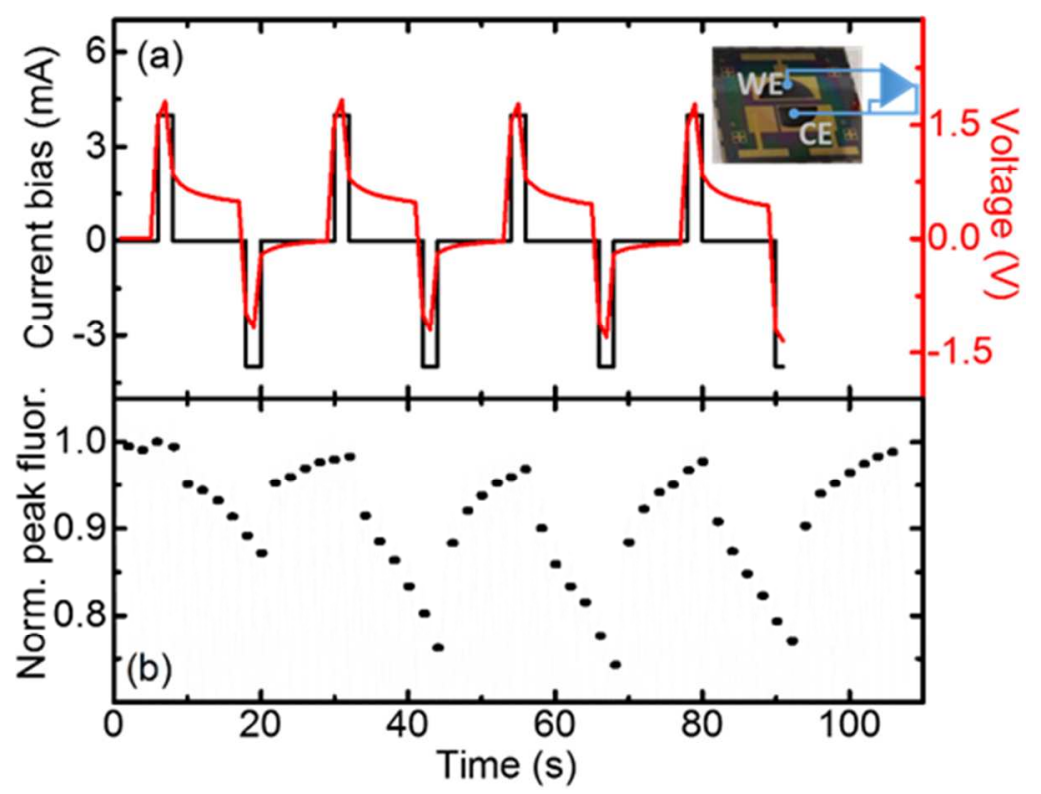


Figure $\mathbf{5}$ (a) Current bias (in black) and voltage response (in red) corresponding to square pulses of $0.4 \mathrm{~mA}$ of amplitude $2 \mathrm{~s}$ of excitation time followed by $10 \mathrm{~s}$ of resting time. (b) Normalised peak fluorescence at the WE during the current application.

\title{
ASSOCIATED CONTENT
}

\section{Supporting Information.}

Supporting information includes notes regarding migration and diffusion of protons, considerations of the approximation of equations 2 and 3, details of the platinisation of the electrodes, details of the resealable microfluidic platform, details of SNARF fluorescence, details of the electropolymerisation in the microfluidic platform, calculation of the minimum $\mathrm{pH}$, and quenching of fluorescence with two electrode configuration.

\section{AUTHOR INFORMATION}

Corresponding Author

Corresponding author: cesar.pascual@list.lu

\begin{abstract}
Author Contributions
The manuscript was written through contributions of all authors. All authors have given approval to the final version of the manuscript.
\end{abstract}

\section{Funding Sources}

FNR ATTRACT project NANOpH.

\section{Notes}

* The decrease of the overall acidity observed during the CV with the closed cell configuration (see the increase of fluorescence from fig 2 (e) to (g)) is opposite to what we observed during the 
polymerisation experiments shown in SI with the cell opened where the overall acidity increased due to electro-polymerisation process (see the SI).

\title{
ACKNOWLEDGMENTS
}

We would like to thank for the useful discussions to Dr. Sivashankar Krishnamoorthy and for their assistance during the fabrication process to Dr. Stephanie Girod, Dr. Pasquale-Antonio Pingue, Dr. Franco Carillo, Dr Daniele Ercolani and Dr. Doriane del Frai. This investigation was financed by the FNR ATTRACT project NANOpH.

\author{
ABBREVIATIONS \\ WE Working electrode \\ CE Counter electrode \\ $\tau$ : characteristic diffusion time for a diffusion barrier \\ $L$ : Length of a Diffusion barrier \\ $D$ : Proton diffusion time \\ $I$ : current \\ $t$ : time \\ $F$ : Faraday constant \\ $V_{\text {cell }}$ : Volume of electrochemical cell \\ $Q$ : Exchanged charge
}

SAM : Self assembled monolayer.

$A_{W E}:$ Area of the working electrode

$S$ : Surface functionalization

$h:$ height of the cell.

4ATP 4-amino-thiol-phenol

SNARF carboxy semi-naphthorhodafluors 
41

44

45 


\section{REFERENCES}

1. Gao.X.; Yu, P.; Sonigo E.L.; Pellois, J.P.; Zhang H, JACS, 1998, 120, 12698-12699.

2. Pellois, J.P.; Zhou, X.; Srivannavit, O.; Zhou, T.; Gulari, E.; and Gao, X, Nat. Biotechnol., 2002, 922-926.

3. Cleland, W.W.; Methods Enzymol., 1982, 87, 390-405.

4. $\quad$ Marangoni, A.G., John Wiley and Sons, Inc. Hoboken, 2003, Chapter 6.

5. Wiedman G.; Kim S.; Zapata-Mercado E.; Wimley WC.; Hristova K., J. Am. Chem. Soc., 2017, 139 (2), 937-945.

6. Maerten, C.; Garnier, T; Lupattelli, P; Chau, N.T.T.; Schaaf, P.; Jierry, L.; Boulmedais, F., Langmuir, 2015, 31, 13385-13393.

7. Egeland, R.D.; Southern, E. M., NSTI-Nanotech, 2004, ISBN 0-9728422-7-6 Vol. 1, 43-49.

8. $\quad$ Egeland, R.D.; Marken, F.; Southern, E. M., Anal. Chem., 2002, 74, 1590-1596.

9. Maurer, K.; Cooper, J.; Caraballo, M.; Crye, J.; Suciu, D.; Ghindilis, A.; Leonetti, J. A.; Wang, W.; Rossi, F.M.; Stöver, A.G.; Larson, C.; Gao, H.; Dill, K.; McShea, A., PLoS ONE 1(1): e34.

10. Chang, Y.; Ahn, Y.S.; Hahn, H.T.; Chen, Y., Langmuir, 2007, 23, 4112-4114.

11. Wang, F.; Liu, X.; Willner, I., Angew. Chem. Int. Ed., 2015, 54, 1098-1129.

12. Elbaz, J.; Wang, F.; Remacle, F.; Willner, I., Nano lett, 2012, 12, 6049-6054.

13. Rydzek, G.; Jierry, L.; Parat, A.; Thomann, J.S.; Voegel, J.C.; Senger, B.; Hemmerl, J.; Ponche, A.; Frisch, B.; Schaaf, P.; Boulmedais, F., Angew. Chem. Int. Ed, 2011, 50, $4374-4377$.

14. Reginald M. Penner, Nat. Chem., 2010, 2, 251-252.

15. Böhm, S.; Olthuis, W.; Bergveld, P., Sens. Actuators B Chem., 1998, 47, 48-53.

16. van der Schoot, B.; Bergveld, P., Sens. Actuators, 1985, 8, 11-22.

17. Olthuis, W.; Bergveld, B., Mikrochim. Acta, 1995, 121, 191-223.

18. Flanagan, T. B.; Oates, W. A., Annu. Rev. Mater. Sci, 1991, 21, 269-304.

19. Frasconi, M.; Tel-Vered, R.; Elbaz, J; Willner, I., J. Am. Chem. Soc., 2010, 132, 20292036.

20. Wang, Y. C.; Lin, C. B.; Su, J. J.; Ru, Y. M.; Wu, Q.; Chen, Z. B.; Mao, B. W.; Tian, Z. W., Anal. Chem., 2011, 83, 4930-4935. 
21. Balakrishnan, D.; Lamblin, G.; Thomann, J. S.; Guillot, J.; Duday, D.; van den Berg, A.; Olthuis, W.; Pascual-Garcia, C., Sci. Rep., 2017, 7, 15401.

22. Ahao, L.B.; Zhang, M.; Ren, B.; Tian, Z. Q.; Wu, D. Y., J. Phys. Chem. C, 2014, 118, 27113-27122.

23. Hayes, W.A.; Shannon, C., Langmuir, 1996, 12, 3688-3694.

24. Wagler, P.F.; Tangen, U.; Maeke, T.; Mc Caskill, J.S., BioSystems, 2012, 109, 2-17.

25. Wiktor, P.; Brunner, A.; Kahn, P.; Qiu, J.; Magee, M.; Bian, X.; Karthikeyan, K.; LaBaer, J., Sci.Rep., 2015, 5, 8736.

26. Chen, S.; Bomer, J.G.; Carlen, E.T.; van den Berg, A., Nano Lett., 2011, 11 , 2334-2341.

27. Clément, N.; Nishiguchi, K.; Dufreche, J.F.; Guerin, D.; Fujiwara, A.; Vuillaume, D., Nano Lett., 2013, 13, 3903-3908.

28. Zong, S.; Wang, Z.; Yang, J.; Cui, Y., Anal. Chem., 2011, 83, 4178-4183.

29. Piotrowski, P.; Wrzosek, B.; Królikowska, A.; Bukowskaa, J., Analyst, 2014, 139, 11011111.

30. Johnson, I.D., Life Technologies Corporation, 2010, 11, Chapter 20.

31. Takulapalli, B. R., et.al., J. Proteome Res., 2012, 11, 4382-4391.

32. Cussler, E. L., Cambridge University press, 1997.

33. Stoychev, D.; Papoutsis, A.; Kelaidopoulou, A.; Kokkinidis, G.; Milchev, A., Mater. Chem. Phy., 2001, 72, 360-365

34. Hsieh, C.T.; Teng, H.; Chen, W.Y.; Cheng, Y.S., Carbon, 2019, 48, 4219-4229.

35. Austin, L.G.; Gagnon, E.G., J. Electrochem. Soc., 1973, 120, 251-254.

36. Li, L.; Qiu, J.; Wang, S., Electrochim. Acta., 2013, 99, 278- 284.

37. Wallace, G.G.; Chen, J.; Mozer, A.J.; Forsyth, M.; MacFarlane, D. R.; Wang, C., Mater. Today., 2009, 12, 20-27.

38. Brousse, T.; Bélanger, D.; Long, J.W., J. Electrochem. Soc., 2015, 162, A5185-A5189. BRIEFS (Word Style "BH_Briefs"). If you are submitting your paper to a journal that requires a brief, provide a one-sentence synopsis for inclusion in the Table of Contents.

SYNOPSIS (Word Style "SN_Synopsis_TOC”). If you are submitting your paper to a journal that requires a synopsis, see the journal's Instructions for Authors for details. 


\section{TABLE OF CONTENTS GRAPHIC}
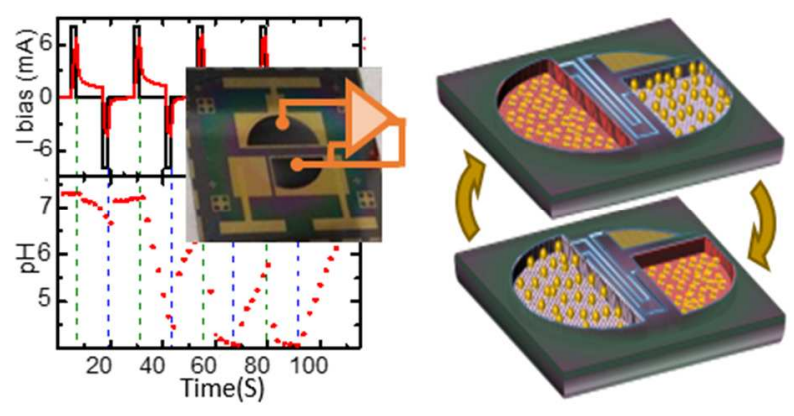


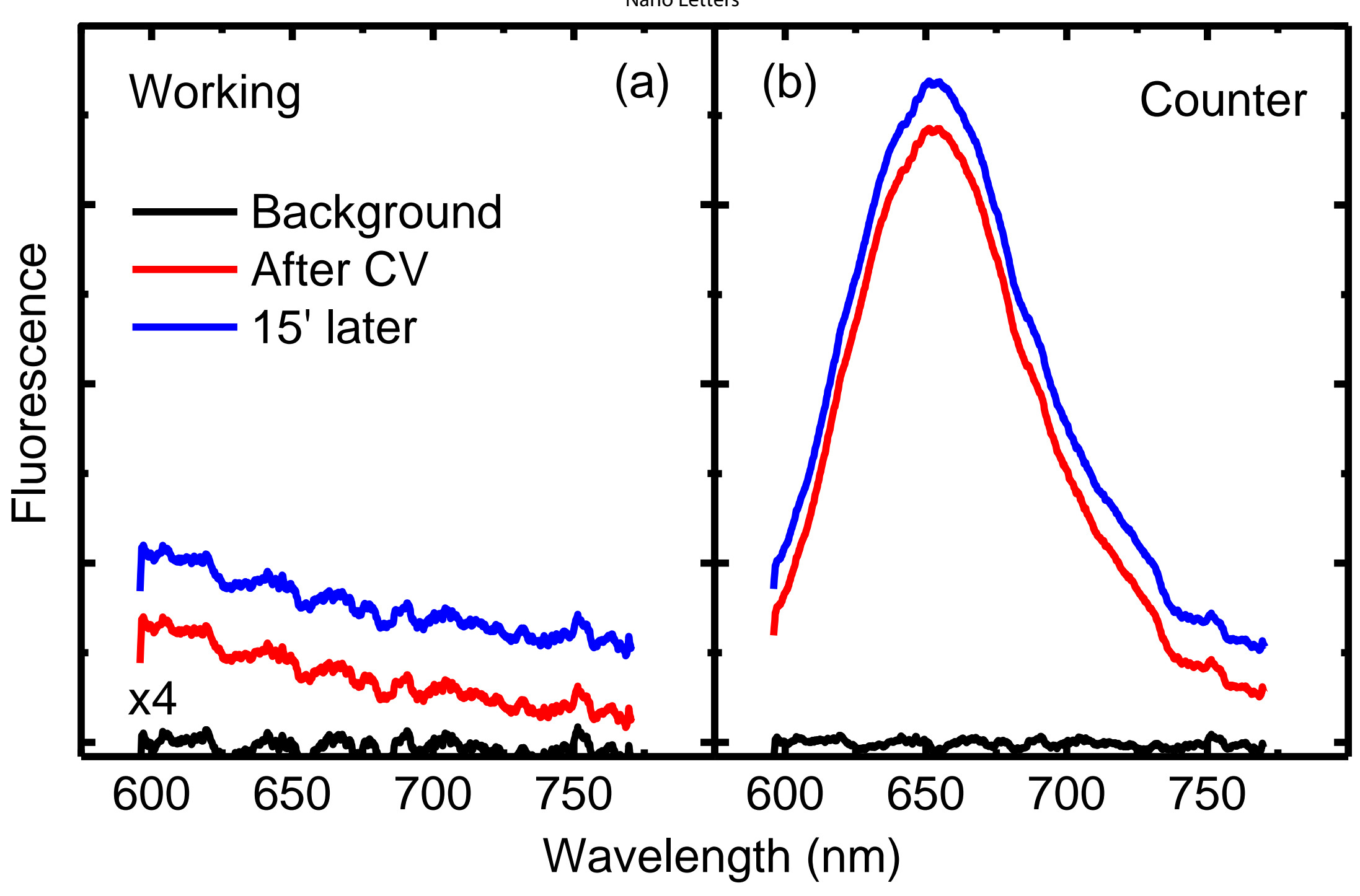




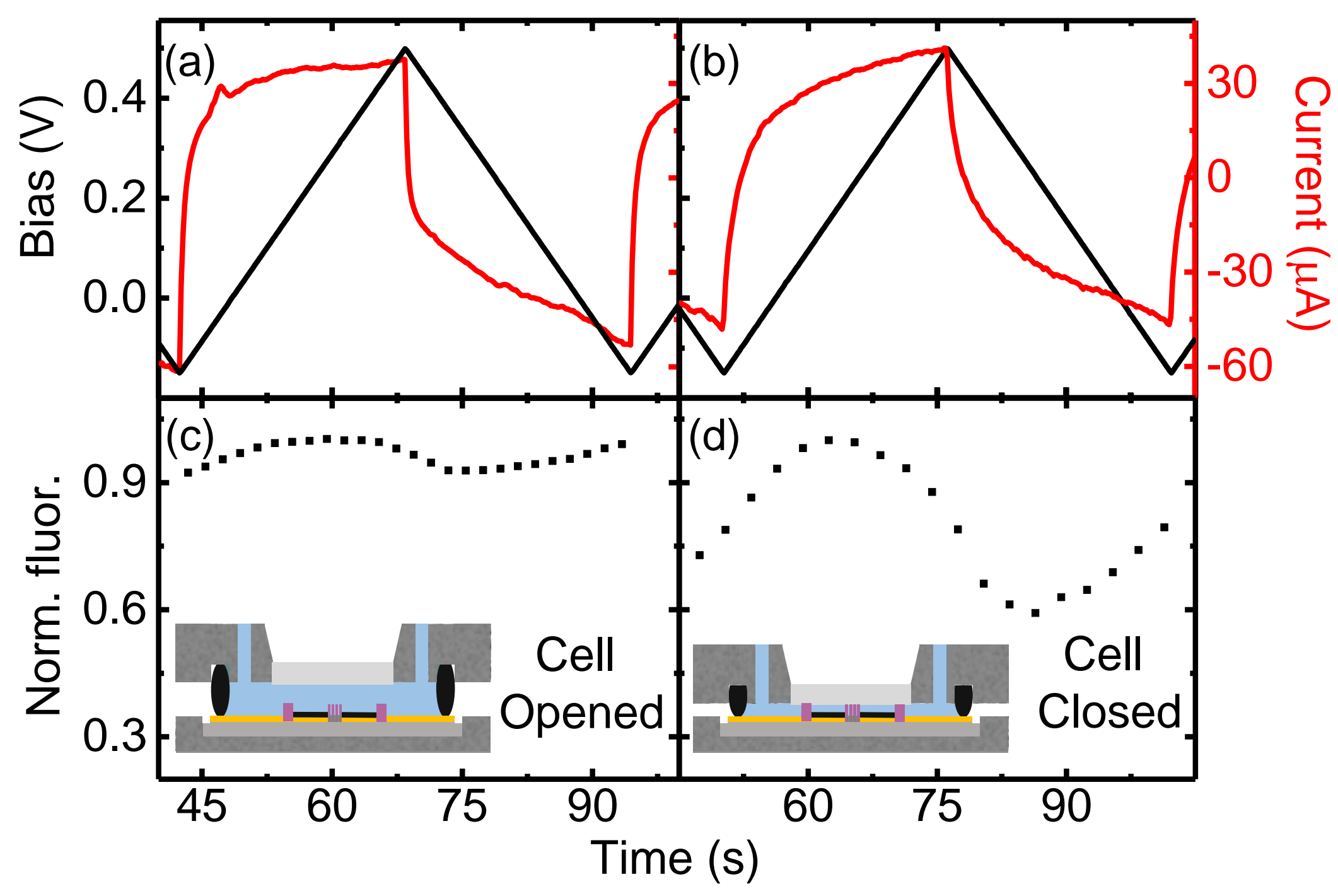




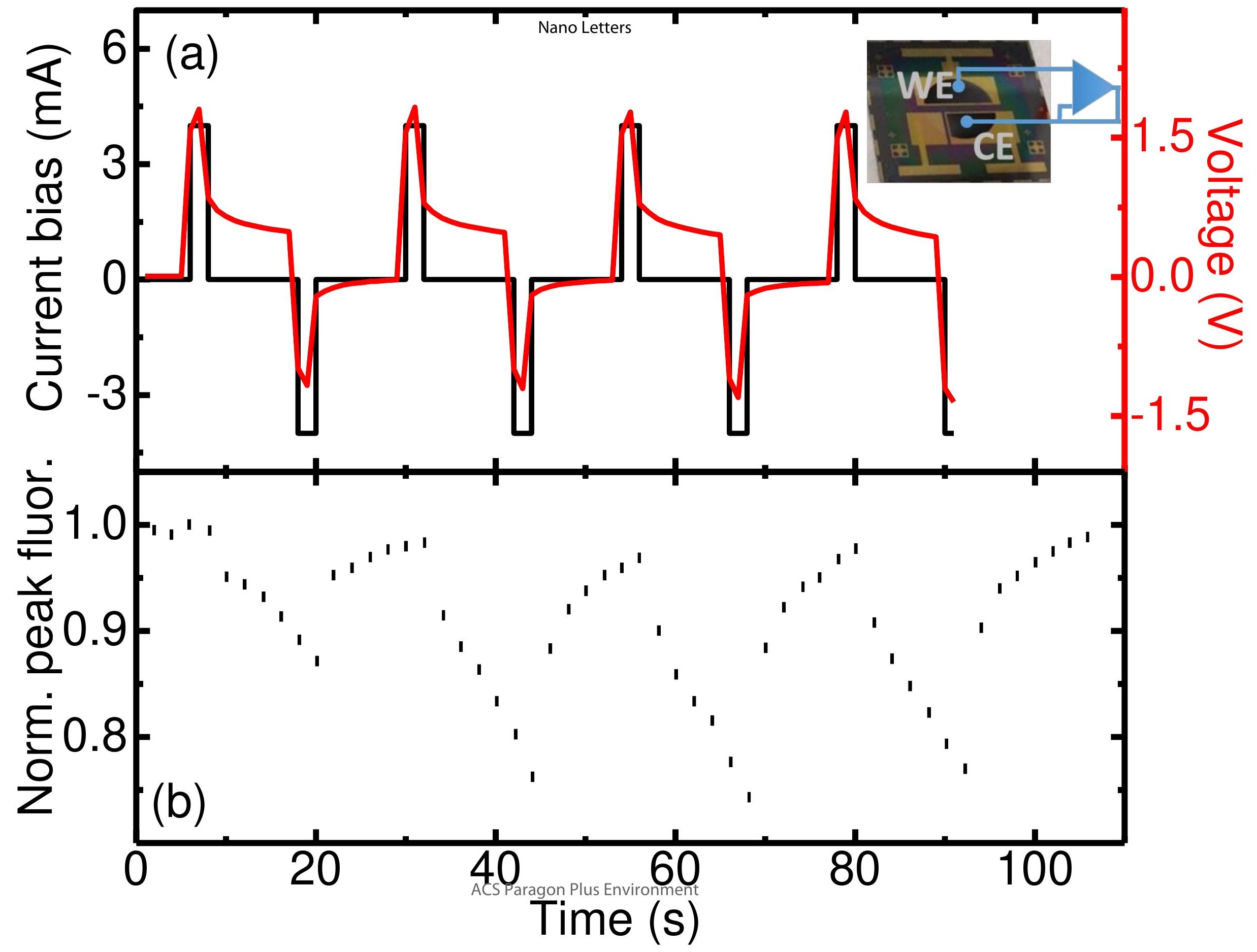

Page 36 of 36 